\title{
Borel convergence of the variational mass expansion
}

\author{
Jean-Loïc Kneur* and Damien Reynaud \\ Physique Mathématique et Théorique, UMR-5825-CNRS, F-34095 Montpellier, France.
}

\begin{abstract}
With an alternative perturbative series, obtained from expansion near a renormalization group "self-consistent mass" solution, in asymptotically free models, the usual factorial behaviour at large expansion orders of certain physical quantities can be improved. This leads to (Borel) convergence of the alternative expansion, at least for particular values, $m_{v} \lesssim 0$, of the new perturbative (mass) parameter. We argue that non-ambiguous estimates of quantities relevant to dynamical (chiral) symmetry breaking in $\mathrm{QCD}$, can be obtained from a direct resummation of this expansion.
\end{abstract}

\section{Introduction}

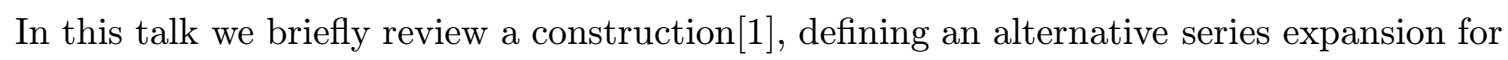
physical Green functions in asymptotically free theories (AFT). The basic idea is to exploit a physically motivated renormalization group (RG) "self-consistent mass" solution, which automatically transforms the ordinary expansion (in a coupling $g$ ) of on-shell Green functions, depending explicitly on a mass $m$, in the form of (mass) power expansions in $(\hat{m} / \Lambda)^{\alpha}$ [ $\hat{m}$ is the renormalization scale-invariant mass, $\Lambda$ the basic RG scale and $\alpha$ is determined by lowest orders RG coefficients]. This resums RG dependence to all orders (at least in specific schemes), and is non-perturbative in the sense that it automatically provides [i, i, a well-defined (analytic) bridge between the usual perturbative regime (corresponding to $\hat{m} \gg \Lambda$ ) and the strongly coupled non-perturbative, massless (chiral) limit (corresponding to $\hat{m} \ll \Lambda$ ). Unfortunately, this is a pure RG result, and its extrapolation to $\hat{m} \rightarrow 0$ turns out to be badly afflicted when taking into account the non-RG perturbative large order behaviour, exhibiting same signs (thus non Borel summable) factorially divergent coefficients

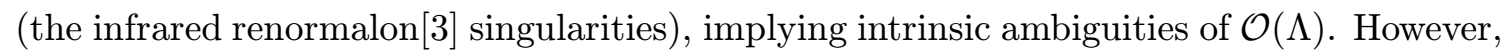
our alternative expansion has the property that it can be smoothly extrapolated down to negative values of the relevant mass expansion parameter, inducing a sign alternation of the coefficients, so that the series is found to be Borel summable[2] near the chirally symmetric, massless limit, which also corresponds to the strongly coupled regime (physical quantities

\footnotetext{
${ }^{*}$ Speaker.
} 
do not depend on the absolute sign of this mass parameter). In addition, the structure of this mass power expansion is such that its convergence properties can be further improved, by combining it with another modification of perturbation theory, known as delta-expansion (DE) or "variationally improved perturbation" (VIP) [i, $\left.\bar{i}_{1}\right]$. The latter is a reorganization of the interaction Lagrangian, involving an arbitrary adjustable parameter fixed by specific (generally optimization) prescriptions. In $D=1$ (oscillator) models, DE-VIP is equivalent to rescaling the adjustable mass with perturbative order [5], which essentially suppresses the factorial asymptotic behaviour of ordinary perturbative coefficients [i, convergent series $\left[\overline{i_{1}}\right]$. Our construction may be considered a field theory generalization of those convergence properties. Resumming this mass power series gives estimates of e.g. the

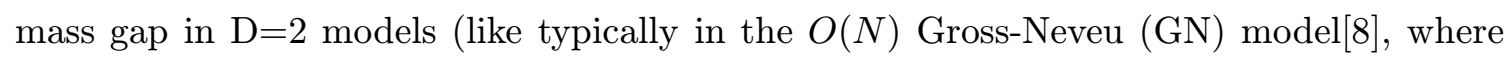
it can be compared to exact results $[9]$ i $)$, with also possible application to more involved theories like QCD, where the non-perturbative chiral symmetry breaking is exhibited by order parameters, accessible from a similar construction [i] in the chiral limit.

\section{Alternative mass expansion and mass gap}

In a renormalization scheme where non-universal (scheme dependent) RG coefficients of $n \geq 3$-loops are set to zero, the pole mass reads [i]

$$
\begin{gathered}
M^{P}(\hat{m})=2^{-C} \hat{m} F^{-A}[C+F]^{-B} \sum_{n=0}^{\infty} d_{n}\left(2 b_{0} F\right)^{-n}, \\
F\left(\frac{\hat{m}}{\Lambda}\right) \equiv \ln \left[\frac{\hat{m}}{\Lambda}\right]-A \ln F-(B-C) \ln [C+F],
\end{gathered}
$$

with $\Lambda$ and $\hat{m}$ the basic scale and RG invariant mass, and $A, B, C$ involve one and two-loop

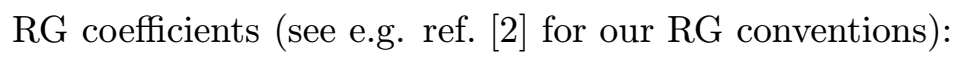

$$
A=\frac{\gamma_{1}}{2 b_{1}}, B=\frac{\gamma_{0}}{2 b_{0}}-A, C=\frac{b_{1}}{2 b_{0}^{2}} .
$$

In $\left(\overline{2} \overline{2}_{-} \overline{1}_{1}\right)$ the coefficients $d_{n}$ essentially include non-RG perturbative contributions from the $n$-loop graphs. At first RG order $\left(b_{1}=\gamma_{1}=0\right)$, Eq. (2.2.23) takes a simpler form

$$
F(\hat{m} / \Lambda) \equiv \ln (\hat{m} / \Lambda)-A_{0} \ln F=A_{0} W\left[A_{0}^{-1}(\hat{m} / \Lambda)^{1 / A_{0}}\right]
$$

where $A_{0}=\frac{\gamma_{0}}{2 b_{0}}$ and the Lambert [10 0 in function $W[x] \equiv \ln x-\ln W$, is plotted in Fig 1 .

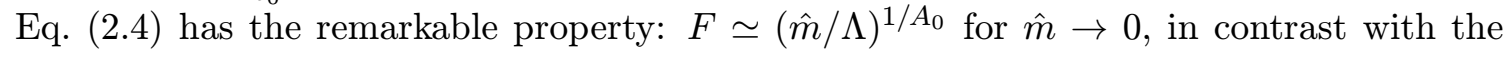
ordinary logarithm, however asymptotic to $F(\hat{m} / \Lambda) \sim\left(2 b_{0} g\right)^{-1}$ for $\hat{m} \gg \Lambda$ (Fig. 1). At

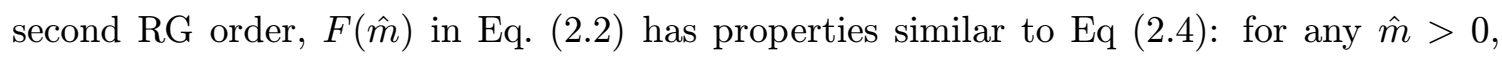
it defines a bridge between the usual short distance perturbative $\hat{m} \gg \Lambda$ (Log) regime, and the non-perturbative $\hat{m} \lesssim \Lambda$ regime, where $F$ has a power expansion. Accordingly, if neglecting (only momentarily) the non-RG $d_{n}$ terms, the pure RG mass $M^{R G}(\hat{m})$ in Eq. $\left(\overline{2} \cdot \overline{2} \cdot \overline{1}^{\prime}\right)$ reads

$$
M^{R G}(\hat{m})=2^{-C} e^{F}[C+F]^{-C} .
$$


This gives a mass gap: $M=(2 C)^{-C} \Lambda$, for $\hat{m} \rightarrow 0$, as easily obtained expanding $(\overline{2} .2 i)$ for $\hat{m} \rightarrow 0$ in $(\overline{2} \cdot \overline{2} \cdot \bar{i})$, equivalently $F \rightarrow 0$ :

$$
M(\hat{m} \rightarrow 0)=(2 C)^{-C} \hat{m}\left[(\hat{m} / \Lambda)^{1 / A}+\cdots\right]^{-A}=(2 C)^{-C} \Lambda\left(1+\mathcal{O}(\hat{m} / \Lambda)^{1 / A}\right),
$$

which may be viewed as a generalization (for $m \neq 0$ ) of dimensional transmutation.

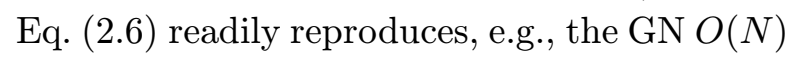
model mass gap [i] in the large $N, m \rightarrow 0$ limit (where $A \rightarrow 1$ for $N \rightarrow \infty$ ). A crucial point is the difference between the usual effective coupling $g\left(p^{2}\right) \equiv 1 /\left[b_{0} \ln \left(p^{2} / \Lambda^{2}\right)\right]$, having a Landau pole at $p^{2}=\Lambda^{2}$, and $F^{-1}(\hat{m})$ here, having its pole at $\hat{m}=$ 0 , governing the massless limit $\left(\overline{2}_{2} . \overline{6}^{\prime}\right)$ of the pure $R G$

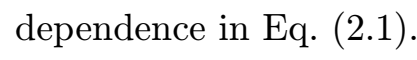

\section{Borel summability near the chiral limit}

Unfortunately, $(\overline{2} . \overline{1})$ differs crucially from the "pure RG" mass, Eqs. ( $\left(\overline{2} . \overline{5}_{1}^{\prime}\right),\left(\overline{2}-\overline{2}^{\prime}\right)$ essentially because the

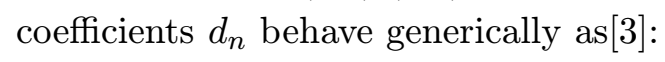

$$
d_{n+1} \sim\left(2 b_{0}\right)^{n} n !
$$

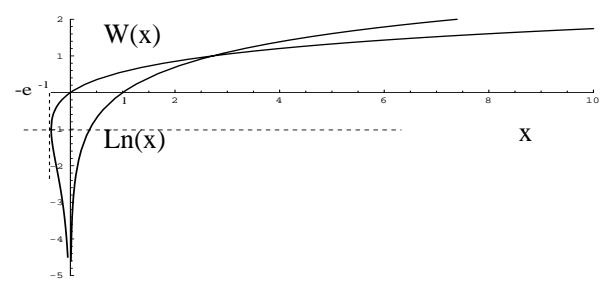

for $n \rightarrow \infty$, so that the series Eq. $\left(\overline{2} \cdot \overline{2}_{1}^{\prime}\right)$ is badly divergent for any $\hat{m}>0$, and not even Borel

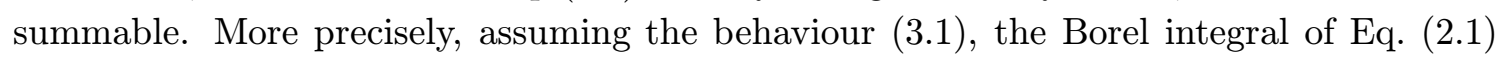
reads:

$$
B I(\hat{m}) \sim 2^{-C} \Lambda e^{F}(C+F)^{-C} \int_{0}^{\infty} d t e^{-t}\left[1+\left(2 b_{0} F\right)^{-1} \sum_{n=0}^{\infty}\left(\frac{t}{F}\right)^{n}\right] .
$$

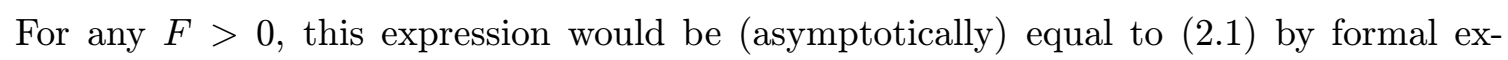
pansion, would the pole at $t=F$ not make the integral (13.2i $)$ ill-defined. One should deform the contour in a non-unique way, going above or below the pole, which leads to an ambiguity $\mathcal{O}\left(e^{-F}\right) \sim \mathcal{O}(\Lambda / \hat{m})$ for the "short distance" $(\hat{m} \gg \Lambda)$ pole mass, in agreement with standard results [3inj. However, (3.2. then $F \equiv-|F|$ simply produces the adequate sign alternation in the factorially growing

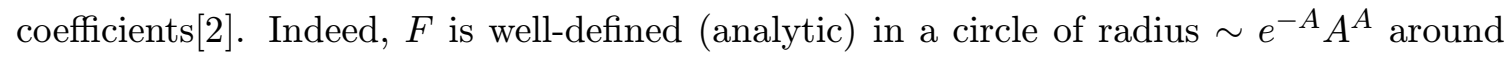
$\hat{m}=0$ and $\operatorname{Re}[F]<0$ for $\hat{m}<0$ (cf Fig. 1, for $A=1$ ). Eq. ( $\left(\overline{3} \cdot \overline{2}^{2}\right)$ for $\operatorname{Re}[F]<0$ gives:

$$
\tilde{M}^{P} / \Lambda \sim e^{-|F|}+\frac{1}{2 b_{0}} \operatorname{Ei}(-|F|)
$$

in terms of the (well-defined) exponential integral function $E i(-x)=-\int_{x}^{\infty} d t e^{-t} / t$ (where for simplicity we neglected in (3.3) the second RG order dependence, irrelevant to large order properties). We obtain in this way Borel convergence for a certain range of the arbitrary mass, strictly only for $R e[\hat{m}]<0$. Moreover, simply from the properties of $F$ around $F \lesssim 0$ the Borel sum (3) the exact $1 / N$ mass gap in the $O(N)$ GN model, which has an explicitly Borel summable perturbative expansion [i] $\left.{ }_{1}^{1} \overline{1}_{1}\right]$. 


\section{Variationally improved mass expansion}

We examine now how to complement the previous construction, based only on RG proper-

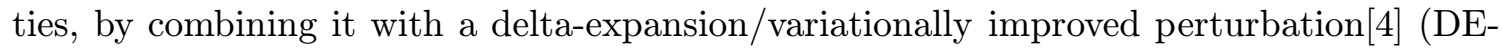
VIP). We define a (linear) DE-VIP as the substitution

$$
m(\mu) \rightarrow(1-\delta) m_{v} ; g^{2}(\mu) \rightarrow \delta g^{2}(\mu)
$$

within perturbative expressions at arbitrary order, where $m(\mu)$ is a (renormalized) Lagrangian mass, $\delta$ the new expansion parameter, and $m_{v}$ an arbitrary adjustable mass.

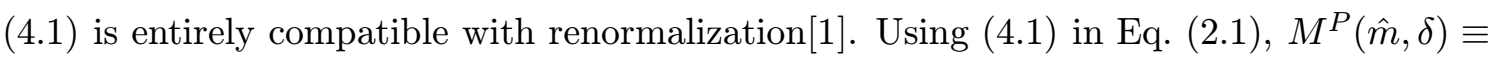
$\sum_{k} a_{k}(\hat{m}) \delta^{k}$ can be most conveniently directly resummed, for $\delta \rightarrow 1$, by contour integration [i] around $\delta=0$, to arbitrary order $K$ : an appropriate change of variable allowing to study the $m(\mu) \rightarrow 0$ (equivalently $\delta \rightarrow 1$ ) limit in Eq. (1). ili) is $\delta \equiv 1-v / K ; \quad m_{v}=K \hat{m}_{v}$. The final contour integral summation gives $[\overline{2}]$ for $K \rightarrow \infty$ :

$$
M^{P} / \Lambda \sim 1+\frac{1}{2 b_{0}} \sum_{q=1}^{N}\left[\sum_{p=0}^{N-q} \frac{\Gamma[p+q](p+q+A)(q+A)^{p-1}}{A^{p} \Gamma[1+p] \Gamma[1+q / A]}\right]\left(m^{\prime \prime}\right)^{-q / A}
$$

(again assuming the leading renormalon behaviour (3.1), with $n \equiv p+q$ ), where $m " \equiv$ $\hat{m}_{v} / \Lambda, N$ is maximal perturbative order, and the contour encircles the semi-axis $\operatorname{Re}[v]<$ 0. Eq. (4) accordingly exhibits a factorial damping of coefficients (as compared to the original perturbative expansion) from the $1 / \Gamma[1+q / A]$ terms, where $A$ in $\left(\overline{2} \cdot \overline{3}^{\prime}\right)$ is schemedependent. Yet, closer examination indicates that the damping is insufficient to make this series readily convergent for $N \rightarrow \infty, m$ " $>0$. Nevertheless, the damping of factorials from appropriate RS choice gives more interesting results, when the series Eq. (4.2) is Borel

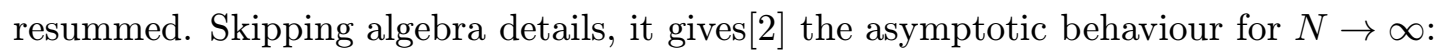

$$
B I_{v a r}\left(m^{\prime \prime}\right) \sim \Lambda\left[1+\int_{0}^{\infty} \frac{d t}{2 b_{0}} \sum_{q}^{\infty} \frac{\left(t^{A} e^{t} / m^{\prime \prime}\right)^{q / A}}{\Gamma[1+q / A]}\right]
$$

so that the Borel integrand in ( $(\overline{4} .3)$ is an entire series (at least for $A>0$ ), with no poles for $0<t<\infty$. The pole at $t_{0}=1$ in the original Borel integrand ( $(12.21)$ was pushed to $t_{0} \rightarrow+\infty$ due to the factorial damping, so that the integral is no longer ambiguous.

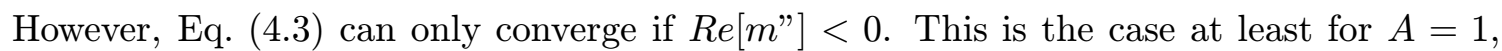
which can always be chosen by a simple scheme change, $\gamma_{1}$ in (2. (2) being scheme dependent. Thus, again one can reach the chiral limit $m " \rightarrow 0$, of main interest, within the Borel-

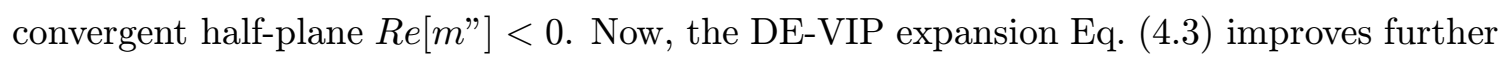
the asymptotic behaviour of the series as compared to (3) at least for $A=1$, due to the extra factorial damping. For $A=1$ and $\operatorname{Re}[m "] \lesssim 0$ Eq. (4' 3 "3) reads

$$
\tilde{M}_{v a r}^{P}(m ") \sim \text { const. } \Lambda(1+f(|m "|))
$$

where $f(|m "|) \rightarrow 0$ exponentially fast for $|m "| \rightarrow 0$ and the unspecified constant includes

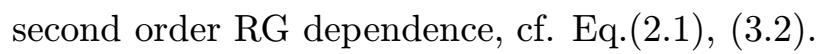




\section{Discussion}

The final DE-VIP result (4.4) is however not completelly independent of the perturbative information, the corresponding series for the asymptotic behaviour being not the exact

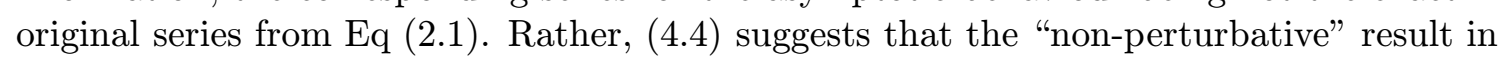
the chiral limit may be essentially determined by pure RG properties, plus eventually the very first few perturbative terms, but not influenced by details of the large perturbative orders. Indeed, renormalon ambiguities are perturbative artifacts expected to disappear (more precisely to cancel out with OPE contributions) in truly non-perturbative

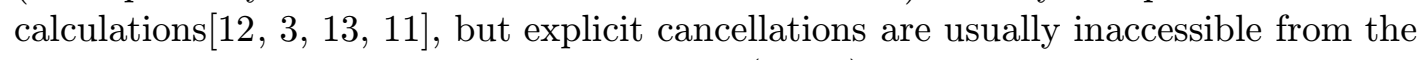
purely perturbative information alone. The (Borel) convergence properties exhibited here

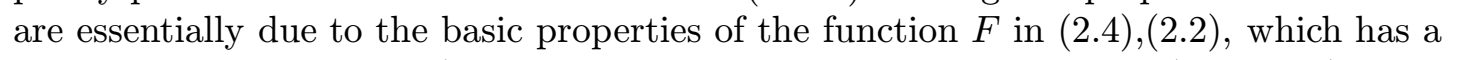
power dependence on $\hat{m}_{v} / \Lambda$, for sufficiently small $\hat{m}_{v}$. Moreover, the (arbitrary) DE-VIP mass parameter $\hat{m}_{v} / \Lambda$ may be rescaled, and/or can take values such as $\operatorname{Re}\left[\hat{m}_{v} / \Lambda\right]<0$, producing sign alternation of factorial coefficients. Our expansion thus appears to "bypass" the need for explicit cancellation between perturbative and non-perturbative contributions, at least for certain physical quantities like the mass gap. Since these formal Borel convergence properties are a priori applicable to any AFT, we argue that it can provide a way to estimate, from direct resummation of this alternative expansion, some of the chiral symmetry breaking order parameters in QCD or other models, which like the mass gap are similarly accessible in the chiral limit from this construction [[1] [1]

\section{References}

[1] C. Arvanitis et al, Int.J.Mod.Phys. A12 (1997) 3307; C. Arvanitis et al, Phys.Lett. B390 (1997) 385; J.-L. Kneur, Phys.Rev. D57 (1998) 2785.

[2] J.-L. Kneur and D. Reynaud, hep-th/0107073.

[3] For a review see M. Beneke, Phys.Rept.317 (1999) 1.

[4] For a review of "delta-expansion" ideas, see e.g. refs. [i] and original references therein.

[5] R. Seznec and J. Zinn-Justin, J. Math. Phys. 20 (1979) 1398.

[6] C.M. Bender and T.T. Wu, Phys. Rev. 184 (1969) 1231; Phys. Rev. D7 (1973) 1620.

[7] A. Duncan and H.F. Jones, Phys. Rev. D47 (1993) 2560; C.M. Bender, A. Duncan and H.F. Jones, Phys. Rev. D 49 (1994) 4219; B. Bellet, P. Garcia and A. Neveu, Int.J.Mod.Phys. A11(1996) 5587; and 5607; C. Arvanitis, H.F. Jones and C. Parker, Phys. Rev. D 52 (1995) 3704; R. Guida, K. Konishi and H. Suzuki, Ann. Phys. 241 (1995) 152; Annals Phys. 249 (1996) 109; H. Kleinert, Phys. Rev. Lett.75 (1995) 2787; W. Janke and H. Kleinert, Phys. Lett. A206 (1995) 283.

[8] D. J. Gross and A. Neveu, Phys. Rev. D10 (1974) 3235.

[9] P. Forgacs, F. Niedermayer and P. Weisz, Nucl. Phys. B 367 (1991) 123; 157.

[10] See e.g. R. Corless et al, Adv. Comput. Math. 5 (1996) 329.

[11] J.-L. Kneur and D. Reynaud, hep-th/0111120.

[12] F. David, Nucl. Phys. B209 (1982) 433; ibid B234 (1984) 237.

[13] M. Beneke, V.M. Braun and N. Kivel, Phys. Lett. B443 (1998) 308. 